\section{Causes of Chronic Retention of Urine in the Primary Care Setting}

Key words: dysuria, overflow incontinence, drug-induced urinary retention

Patients with dysuria and frequent urination owing to chronic retention of urine (CRU), as defined by the International Continence Society (1), are sometimes seen in primary care settings, especially in practices that treat numerous aged patients. The main causes of CRU include diabetes mellitus, prostatic enlargement, herniated intervertebral disc, stroke, and certain drugs $(2,3)$. To our knowledge, however, there have been few detailed descriptions of the causes of CRU, particularly drug-induced forms, in the primary care population. Here, we examined the causes of incontinence in this setting.

One hundred consecutive patients (67 outpatients and 33 inpatients) with definitive CRU who were referred to Nishiagatsuma Welfare Hospital from February 2002 to August 2004 were included in the study. All of the patients complained of dysuria and frequent urination. The diagnosis in all cases was based on ultrasonography or urethral catheterization demonstrating that the post-void residual volume exceeded the voiding volume. The patients included 65 men and 35 women. The mean age was $74 \pm 11$ (SD) years.

The underlying cause of CRU was examined in each patient. Although the cause of CRU remained unknown for some patients, the most common cause was found to be prostate disease (23 cases), followed by brain disorders (Table 1). Other causes of CRU in this population included diabetes, spinal degenerative diseases, and incontinence following surgery for pelvic or femoral neck fracture. A total of 12 patients had CRU attributable to the side effects of various medicines, including propiverine (6 cases), amytriptyline (2 cases), common cold medicines (2 cases), oxybutynin (1 case), and eperisone (1 case). In patients with drug-induced CRU, the symptoms started when the intake of the corresponding medication began and improved soon after cessation of the medication.

When examining patients with CRU in primary care practice, physicians must seek an underlying condition, such as diabetes, prostate disease, spinal disease, or medication use $(2,3)$. The underlying diseases seen in the present study were consistent with the generally accepted causes, although the frequencies of these conditions as causes of CRU have not been reported (2). Drug-induced CRU has been reported, and certain drugs are known to be associated with CRU (4, 5 ), although the role of medication as a cause of overflow incontinence has not been discussed in the literature. The anticholinergic, relaxant, psychotropic, and cold medications that were found to be possible causes of CRU in this study may act through interference with bladder contraction (4). For example, whereas bladder contraction requires cholinergic parasympathetic innervation coordinated with sphincter relaxation, the antimuscarinic effects of some anticholinergic drugs can reduce the $\alpha$-adrenoceptor input from the sympathetic nervous system (4). These drugs are commonly prescribed by internists.

Drug-induced CRU might be more frequent than our findings indicate, because mild or transient incontinence (e.g., that caused by common cold medications) might be overlooked or go unreported by the patient. In addition, elderly patients are often asymptomatic and tend to suffer from the side effects of drugs at a higher frequency than do younger patients. It is difficult to anticipate drug-induced CRU owing to its idiosyncratic occurrence (4). If a bladder outflow obstruction or residual volume after voiding is pre-existing, drug-induced CRU is more likely to manifest. Before prescribing drugs such as those listed above, physicians should consider whether ultrasonographic evidence of residual volume has been observed. As drug-induced CRU is iatrogenic, physicians must consider medication as a possible cause when examining patients with dysuria and urinary frequency

Table 1. Underlying Causes of Chronic Retention of Urine in 100 Primary Care Patients

\begin{tabular}{lc}
\hline \multicolumn{1}{c}{ Category } & $\begin{array}{c}\text { Number of cases } \\
(\mathrm{n}=100)\end{array}$ \\
\hline Prostate disease & 25 \\
Brain disorders & 22 \\
Unknown causes & 19 \\
Side effects of medications & 12 \\
Following surgery for pelvic or femoral neck fractures & 8 \\
Diabetes mellitus & 7 \\
Degenerative spinal diseases & 7 \\
\hline
\end{tabular}


in the primary care setting.

\section{Gotaro Kurasawa, Kazuhiko Kotani*, Miwa KURASAWA**, Noriaki TAKAMA** and Kenichiro ORIMO**}

From Departments of Urology and **Internal Medicine, Nishiagatsuma Welfare Hospital, Naganohara and *Division of Health Administration and Promotion, Faculty of Medicine, Tottori University, Yonago

Received for publication October 27, 2004; Accepted for publication February 16, 2005

Reprint requests should be addressed to Dr. Gotaro Kurasawa, Department of Urology, Nishiagatsuma Welfare Hospital, 746-4 Ohtsu, Naganohara, Agatsuma, Gunma 377-1308

\section{References}

1) Abrams P, Cardozo L, Fall M, et al. The standardisation of terminology of lower urinary tract function: Report from the Standardisation Subcommittee of the International Continence Society. Neurourol Urodyn 21: $167-178,2002$.

2) Peggs JF. Urinary incontinence in the elderly: Pharmacologic therapies. Am Fam Physician 46: 1763-1769, 1992.

3) Finkelstein MM. Medical conditions, medications, and urinary incontinence: Analysis of a population-based survey. Can Fam Physician 48: 96-101, 2002.

4) Drake MJ, Nixon PM, Crew JP. Drug-induced bladder and urinary disorders. Incidence, prevention and management. Drug Safety 19: 45-55, 1998.

5) Uozumi J. Drug-induced urinary retention and its management. Journal of Integrated Medicine (JIM) 11: 421-423, 2001 (in Japanese). 